\title{
Tempo de prática de atividade física no lazer como fator discriminatório da ausência de excesso de peso corporal
}

\author{
Physical activity time at leisure as discriminatory factor in the absence of \\ excess body weight
}

Thiago Ferreira de Sousa'; Aline Rodrigues Barbosa²; Francisco Teixeira Coelho ${ }^{3}$

\begin{abstract}
Resumo
O objetivo deste estudo foi selecionar, por meio de três inquéritos transversais com universitários, os melhores pontos de corte dos minutos de prática de atividade física aeróbica no lazer, de intensidade moderada a vigorosa, como discriminador da ausência do excesso de peso corporal. Os inquéritos foram realizados nos anos de 2010, 2012 e 2014, com estudantes de uma universidade do estado da Bahia, com média etária de 24 anos $(\mathrm{dp}=5,8)$ e $51,5 \%$ do sexo feminino. A faixa de peso corporal recomendável foi (índice de massa corporal de $18,5 \mathrm{~kg} / \mathrm{m}^{2}$ a 24,9 $\mathrm{kg} / \mathrm{m}^{2}$ ) empregada como categoria de classificação e a variável testada foram os minutos de atividades físicas aeróbicas de intensidade moderada a vigorosa praticadas no lazer. As estimativas dos pontos de corte foram analisadas pelas curvas ROC (Receiver Operating Characteristic), para todos os universitários e estratificadas para as categorias das variáveis sociodemográficas e dos hábitos alimentares. O número de participantes em cada inquérito foi de 947 em 2010, 940 em 2012 e 915 em 2014. A prevalência de peso recomendável foi de $73,1 \%$ e a média dos minutos de atividades físicas no lazer foi de 270,1 (dp: 731,4; mediana de 0; variação interquartil: 360). De modo geral, a área sob a curva ROC foi de 0,56 (IC95\%: 0,54 - 0,58), com ponto de corte de 150 minutos. A capacidade dos minutos de atividades físicas no lazer para a predição da ausência de excesso de peso corporal foi baixa e o ponto de corte que melhor discriminou essa característica foi de 150 minutos por semana.
\end{abstract}

\section{Palavras-chave}

Sobrepeso; Obesidade; Estudantes; Estudos transversais.

\begin{abstract}
The aim of this study was to select, through of the analysis of three cross-sectional surveys with college, the best cutoffs of minutes of practice of aerobic physical activity during leisure-time in moderate to vigorous intensities, as discriminator of the absence of excess body weight (overweight and obesity). The surveys were realized in the years of 2010, 2012 and 2014 in university from state Babia, with mean age of 24 years $(S D=5,8)$ and $51.5 \%$ of female. The recommended body weight range (body mass index of $18.5 \mathrm{~kg} / \mathrm{m}^{2}$ to $24.9 \mathrm{~kg} / \mathrm{m}^{2}$ ) was used as classification category and the variable tested were the minutes of physical activity in leisure, aerobic, in moderate to vigorous intensities. Estimates of cutoffs were analyzed by ROC (Receiver Operating Characteristic) curves, general and stratified of according categories of sociodemographic and eating habits variables. The number of participants in each surveys were: 947 in 2010, 940 in 2012 and 915 in 2014. The prevalence of recommended body weight was $73.1 \%$ and the average minutes of physical activity in leisure-time was 270.1 (SD: 731.4; median: 0 ; interquartile range: 360 ). The best cut-off points, in general, was 150 minutes (ROC: 0.56; CI95\%: $0.54-0.58$ ). The capacity of leisure-time physical activities minutes to predict the absence of excess body weight was low and the cutoff better discriminated that characteristic was 150 minutes per week.
\end{abstract}

\section{Keywords}

Overweight; Obesity; Students; Cross-sectional surveys.

\section{Introdução}

O excesso de peso corporal (sobrepeso e obesidade)

1 Universidade Federal do Recôncavo da Bahia, Centro de Formação de Professores, Amargosa, Bahia, Brasil.

2 Programa de Pós-Graduação em Educação Física da Universidade Federal de Santa Catarina, Centro de Desportos, Florianópolis, Santa Catarina, Brasil.

3 Universidade Federal do Triângulo Mineiro, Departamento de Ciências do Esporte, Uberaba, Minas Gerais, Brasil. representa um dos principais problemas de saúde pública em nível mundial ${ }^{1,2}$. A Organização Mundial de Saúde apontou que em 2014, no mundo, a prevalência desse índice, em adultos, foi de $52 \%^{3}$, e que poderá acometer cerca de 3,28 bilhões de pessoas em $2030^{4}$. Em universitários brasileiros, as prevalências do excesso de peso corporal, verificada em pesquisas 
em diferentes regiões, variaram de $16 \%$ a $27,4 \%^{5-8}$.

A prática de atividades físicas (AF) estão entre as ações direcionadas à prevenção do excesso de peso corporal $^{9-11}$. O American College of Sports Medicine (ACSM) sugere que a prática de 150 a 250 minutos por semana de AF de intensidade moderada seja suficiente para prevenir o aumento de peso corporal ${ }^{9}$. Contudo, quando a intenção é a diminuição de peso corporal, maiores quantidades de AF (>250 minutos por semana) têm sido recomendadas'.

As recomendações específicas para a prevenção do aumento de gordura corporal consideram a prática de $\mathrm{AF}$ em todos os domínios (trabalho, doméstico, deslocamento e lazer) $)^{9-11}$ e especificamente para o domínio do lazer, ainda não está estabelecida a quantidade de minutos necessários à prevenção do excesso de peso corporal. A caracterização da quantidade mínima dos minutos de AF no lazer poderão contribuir para a identificação ou ajuste das orientações sobre a quantidade essencial para a prevenção desse risco à saúde. Sendo assim, o objetivo deste estudo foi selecionar, por meio da análise de três inquéritos transversais com universitários de uma instituição de ensino superior, os melhores pontos de corte dos minutos de prática de AF aeróbicas de intensidade moderada a vigorosa, praticadas no lazer, como discriminador da ausência do excesso de peso corporal.

\section{Métodos}

Este estudo é proveniente dos três inquéritos do estudo MONISA (Monitoramento dos Indicadores de Saúde e Qualidade de Vida em Acadêmicos) executado em uma instituição de ensino superior do estado da Bahia, Brasil. O detalhamento metodológico foi apresentado em estudo prévio $^{12}$. O comitê de ética em pesquisa com seres humanos aprovou o desenho metodológico deste estudo, sob número de protocolo 382 do ano de 2010.

As populações alvo foram os universitários matriculados no segundo semestre letivo da instituição, nos anos de 2010, 2012 e 2014. Os estudantes dos programas de ensino a distância, aqueles com matrícula especial e os ingressantes no segundo semestre letivo não foram incluídos na população alvo.

Os cálculos amostrais consideraram, nos três inquéritos, a quantidade de universitários na população alvo, prevalência dos desfechos em $50 \%$, erro máximo aceitável de três pontos percentuais e nível de confiança de $95 \%{ }^{13}$. Foram incluídos acréscimos de $20 \%$ para possíveis perdas e recusas, e mais $15 \%$ para a análise de estudos de associação, respectivamente. As populações alvo em cada ano dos inquéritos foram: ano 2010, 5.461; ano 2012, 5.767; ano 2014, 5.224. As amostras estimadas para a participação foram de 1.232 em 2010, 1.243 em 2012 e 1.223 em 2014.

As amostras foram estratificadas de acordo com a distribuição da população alvo nos cursos (30 cursos no inquérito de 2010; 34 cursos em 2012; e 33 cursos em 2014, em virtude da junção dos cursos de Letras com habilitação em espanhol e Letras com habilitação em inglês), nos períodos de estudo (diurno e noturno) e nos anos de ingresso na instituição. Para os anos de entrada na universidade, foram criados quatro estratos, tendo em vista o mínimo de quatro anos para a conclusão na maioria dos cursos, sendo: no inquérito de 2010 os anos de 2010, 2009, 2008 e 2007 ou anteriores; no inquérito de 2012 os anos de 2012, 2011, 2010 e 2009 ou anteriores; e no inquérito de 2014 os anos de 2014, 2013, 2012 e 2011 ou anteriores. Por fim, os universitários foram escolhidos por processo de seleção aleatória simples, em cada estrato, por meio da lista de matrícula em ordem alfabética.

Os treinamentos com as equipes de coleta de dados, compostas por professores e universitários da instituição, não pertencentes as amostras, foram realizados 
nos meses de julho e agosto. As coletas de dados foram realizadas nos meses de setembro a novembro. Os universitários foram procurados na instituição em até três tentativas, em dias e horários diferentes. Caso não fossem encontrados ou recusassem a participação, não foi realizada a substituição.

$\mathrm{Na}$ coleta de dados foi utilizado o questionário ISAQ-A (Indicadores de Saúde e Qualidade de Vida em Acadêmicos), elaborado para a aplicação em estudos epidemiológicos com universitários ${ }^{14}$. A variável de classificação deste estudo foi o índice de massa corporal (IMC), calculado pela equação padrão (massa corporal dividido pela estatura ao quadrado), por meio das medidas referidas da massa corporal e estatura. Os níveis de validade de utilização das informações referidas da massa corporal e estatura, e do IMC cálculo com base nessas medidas referidas, em universitários, estabelecidos via gráfico de dispersão de Bland-Altman são aceitáveis ${ }^{15}$. Os níveis de sensibilidade (80\%) e especificidade $(95,5 \%)$ do excesso de peso corporal, calculado por meio das medidas referidas, em comparação ao excesso de peso corporal calculado com base as medidas aferidas são satisfatórias ${ }^{15}$.

A variável foi classificada de acordo com os critérios da Organização Mundial de Saúde ${ }^{16}$, para adultos de 18 a 59 anos, em peso recomendável o IMC de 18,5 kg/ $\mathrm{m}^{2}$ a $24,9 \mathrm{~kg} / \mathrm{m}^{2}$ e excesso de peso corporal o IMC $\geq 25 \mathrm{~kg} / \mathrm{m}^{2}$. Os universitários com idade inferior a 18 anos foram excluídos, em virtude da aplicação de outros critérios de categorização do excesso de peso corporal e optou-se por excluir aqueles classificados com baixo peso $\left(\leq 18,4 \mathrm{~kg} / \mathrm{m}^{2}\right)$ devido à baixa frequência nos inquéritos (2010: 8,6\%; 2012: 8,7\%; 2014: 7,9\%). Após isso foi atribuída a seguinte classificação: faixa de peso recomendável codificada como 1 e o excesso de peso corporal como 0 .

A variável testada foram os minutos de $\mathrm{AF}$, aeróbicas, nas intensidades moderada a vigorosa, praticadas no lazer, obtidos via questionário ISAQ-A, que apresenta níveis satisfatórios de reprodutibilidade para os minutos de AF praticadas no lazer, mediante análise do gráfico de dispersão de Bland-Altman ${ }^{14}$. O produto dos dias pelos minutos de prática por dia para cada uma das opções das modalidades foi somado. A intensidade das atividades foi obtida por meio do compêndio, considerando apenas aquelas classificadas como moderadas (3 a 5,9 METS) e vigorosas $(\geq 6 \mathrm{METS})^{17}$. Os minutos das modalidades vigorosas foram multiplicados por dois ${ }^{18,19}$. As AF moderadas foram: ciclismo, voleibol, natação, ginástica em geral, caminhada em esteira, hidroginástica, surfe e caminhada em locais abertos; e as $\mathrm{AF}$ vigorosas foram: futebol, basquetebol, handebol, corrida em locais abertos, ginástica aeróbica, lutas ou artes marciais, tênis e corrida em esteira ${ }^{14}$.

As variáveis sociodemográficas e referentes aos hábitos alimentares foram: sexo, faixa etária (18 a 24 anos, 25 a 34 anos e 35 anos ou mais), consumo de frutas em dias de uma semana típica (até 4 dias por semana e $\geq 5$ dias por semana) ${ }^{20}$, consumo de hortaliças em dias de uma semana típica (até 4 dias por semana e $\geq 5$ dias por semana $)^{20}$, consumo de carne vermelha gordurosa, de boi, porco ou cabrito (não: nenhum dia por semana; sim: $\geq 1$ dia por semana) ${ }^{20}$, consumo de frango com gordura, mediante o relato de consumo de frango sem remover a gordura visível na pele (não: nenhum dia por semana; sim: $\geq 1$ dia por semana) ${ }^{20}$.

As informações foram tabuladas no software EpiData versão 3.1 e as análises realizadas no SPSS versão 15.0. Foram utilizadas análises descritivas de proporção, média, desvio padrão (dp), mediana, variação interquartil, mínimo e máximo valores. O teste qui-quadrado para tendência linear foi utilizado para analisar a associação entre as variáveis sociodemográficas, hábitos alimentares e IMC em relação aos anos dos inquéritos e o teste Kruskall-Wallis para a comparação das medianas dos minutos de AF no lazer entre os inquéritos, para fins de caracterização da amostra. 
Para discriminar o poder e os pontos de corte dos minutos de AF no lazer para a predição da ausência do excesso de peso corporal foi utilizado às curvas ROC (Receiver Operating Characteristic) e identificadas às áreas abaixo das curvas ROC e os intervalos de confiança a 95\% (IC95\%). As áreas com valores >0,50 e limite inferior do IC95\% >0,50 foram consideradas como discriminatórias para a estimativa de pontos de corte. Níveis de sensibilidade e especificidade para os pontos de corte foram estimados e os pontos que melhor discriminaram a ausência de excesso de peso corporal foram aqueles que apresentaram equilíbrio entre essas duas características ${ }^{21}$. As análises foram realizadas para todos os universitários e estratificadas pelas categorias das variáveis sociodemográficas e dos hábitos alimentares para as amostras dos três inquéritos simultaneamente. O nível de significância adotado foi de 5\%.

\section{Resultados}

Participaram deste estudo em cada inquérito 1.084, 1.085 e 1.041 universitários, nos anos de 2010, 2012 e 2014, respectivamente. Com a exclusão dos universitários com idade até 17 anos e com baixo peso, a amostra de universitários foi de 2.802 na soma dos três inquéritos (média de idade de 24 anos; dp: 5,8; 18 a 57 anos), com a participação de 947 em 2010 (média de idade de 23,7 anos; dp: 5,3; 18 a 52 anos), 940 em 2012 (média de idade de 24,3 anos; dp: 6,1; 18 a 54 anos) e 915 em 2014 (média de idade de 23,9 anos; dp: 5,9; 18 a 57 anos). A Tabela 1 apresenta a caracterização da amostra de universitários de modo geral e em cada inquérito. Observou-se predomínio das mulheres e universitários da faixa etária de 18 a 24 anos. Houve maioria de universitários que informaram consumir frutas em até quatro dias por semana, bem como o consumo de frango com gordura e salgados. A prevalência de universitários na faixa de peso recomendável foi de $73,1 \%$, com diminuição significativa das prevalências ao longo dos inquéritos (Tabela 1).

TABELA 1 - Descrição das variáveis sociodemográficas, dos hábitos alimentares e do índice de massa corporal dos universitários. Bahia. 2010, 2012 e 2014.

\begin{tabular}{|c|c|c|c|c|c|}
\hline \multirow{2}{*}{ Variáveis } & Geral & 2010 & 2012 & 2014 & \multirow{2}{*}{$p^{*}$} \\
\hline & (n) $\%$ & (n) $\%$ & (n) $\%$ & (n) $\%$ & \\
\hline Sexo & & & & & 0,68 \\
\hline Masculino & (1.357) 48,5 & (457) 48,3 & (449) 47,8 & (451) 49,3 & \\
\hline Feminino & (1.443) 51,5 & (489) 51,7 & (490) 52,2 & (464) 50,7 & \\
\hline Faixa etária & & & & & 0,93 \\
\hline 18 a 24 anos & (1.950) 69,6 & $(671) 70,9$ & $(625) 66,5$ & (654) 71,5 & \\
\hline 25 a 34 anos & (685) 24,4 & (226) 23,9 & (249) 26,5 & (210) 23,0 & \\
\hline 35 anos ou mais & (167) 6,0 & (50) 5,3 & (66) 7,0 & (51) 5,6 & \\
\hline Consumo de frutas & & & & & $<0,01$ \\
\hline Até 4 dias por semana & (2.161) 78,0 & (755) 81,3 & (740) 79,6 & (666) 73,2 & \\
\hline 5 ou mais dias por semana & (608) 22,0 & (174) 18,7 & (190) 20,4 & (244) 26,8 & \\
\hline Consumo de hortaliças & & & & & 0,02 \\
\hline Até 4 dias por semana & (1.508) 55,3 & (531) 57,4 & (509) 56,3 & (468) 52,1 & \\
\hline 5 ou mais dias por semana & (1.220) 44,7 & (394) 42,6 & (395) 43,7 & (431) 47,9 & \\
\hline Consumo de salgados & & & & & $<0,01$ \\
\hline Não & (315) 11,7 & (91) 9,8 & (97) 10,9 & (127) 14,3 & \\
\hline Sim & $(2.384) 88,3$ & (833) 90,2 & (792) 89,1 & (759) 85,7 & \\
\hline Consumo de carnes com gordura & & & & & 0,87 \\
\hline Não & (244) 9,0 & (83) 9,0 & (79) 8,8 & (82) 9,2 & \\
\hline Sim & (2.463) 91,0 & (840) 91,0 & (815) 91,2 & (808) 90,8 & \\
\hline
\end{tabular}


... continua

Consumo de frango com gordura

\begin{tabular}{lcclll} 
Não & $(1.317) 48,5$ & $(481) 51,8$ & $(421) 46,9$ & $(415) 46,7$ & \\
Sim & $(1.396) 51,5$ & $(447) 48,2$ & $(476) 53,1$ & $(473) 53,3$ & \\
Índice de Massa Corporal & & & & & $<0,01$ \\
$\begin{array}{l}\text { Peso recomendável } \\
\text { Com excesso }\end{array}$ & $(2.049) 73,1$ & $(715) 75,5$ & $(693) 73,7$ & $(641) 70,1$ & \\
\hline
\end{tabular}

*p valor do teste Qui-quadrado para tendência linear; \%: Proporção.

A média dos minutos de AF no lazer, de modo geral, foi de 270,1 (dp: 731,4; mediana de 0; variação interquartil: 360), em 2010 foi de 227,2 (dp: 447,9; mediana: 0; variação interquartil: 300), em 2012 foi de 278,7 (dp: 795,6; mediana: 0; variação interquartil: 360) e em 2014 foi de 305,0 (dp: 879,0; mediana: 0; variação interquartil: 360). A mediana dos minutos de AF no lazer não diferiram entre os inquéritos ( $p>0,05)$.

As áreas sob a curva ROC são apresentadas na Tabela 2. De modo geral, a área sob a curva ROC dos minutos de AF lazer foi de 0,56 (IC95\%: 0,53 - 0,58). As áreas das seguintes características dos universitários foram maiores que o critério mínimo deste estudo: sexo masculino, universitários de 18 a 24 anos e de 35 anos ou mais, consumo de frutas até quatro dias por semana, consumo de hortaliças até quatro dias por semana e cinco ou mais dias por semana, consumo de salgados, não consumir e consumir carnes com gordura, e não consumir e consumir frango com gordura.

TABELA 2 - Descrição das áreas sob a curva ROC, pontos de corte, níveis de sensibilidade e especificidade dos minutos de AF no lazer como discriminador da ausência de excesso de peso corporal em universitários*. Bahia. 2010, 2012 e 2014.

\begin{tabular}{|c|c|c|c|c|}
\hline Variáveis & ROC (IC95\%) & PC & Sens. \% (IC95\%) & Esp. \% (IC95\%) \\
\hline Geral & $0,56(0,54-0,58)$ & 150 & $64,9(62,8-67,0)$ & $45,6(42,0-49,3)$ \\
\hline \multicolumn{5}{|l|}{ Sexo } \\
\hline Masculino & $0,54(0,51-0,57)$ & 670 & $83,9(81,3-86,2)$ & $24,6(20,8-28,7)$ \\
\hline Feminino & $0,53(0,49-0,57)$ & NR & - & - \\
\hline \multicolumn{5}{|l|}{ Faixa etária } \\
\hline 18 a 24 anos & $0,57(0,54-0,60)$ & 155 & $65,3(62,8-67,6)$ & $47,1(42,2-52,0)$ \\
\hline 25 a 34 anos & $0,52(0,48-0,57)$ & NR & - & - \\
\hline 35 anos ou mais & $0,62(0,53-0,70)$ & 150 & $73,8(62,1-83,0)$ & $49,5(39,5-59,4)$ \\
\hline \multicolumn{5}{|l|}{ Consumo de frutas } \\
\hline Até 4 dias por semana & $0,56(0,53-0,59)$ & 155 & $66,7(64,3-69,0)$ & $44,7(40,6-48,9)$ \\
\hline 5 ou mais dias por semana & $0,54(0,49-0,59)$ & NR & - & - \\
\hline \multicolumn{5}{|l|}{ Consumo de hortaliças } \\
\hline Até 4 dias por semana & $0,55(0,52-0,59)$ & 155 & $65,7(62,8-68,4)$ & $45,3(40,4-50,3)$ \\
\hline 5 ou mais dias por semana & $0,57(0,53-0,60)$ & 287 & $73,3(70,3-76,2)$ & $39,0(33,9-44,3)$ \\
\hline \multicolumn{5}{|l|}{ Consumo de salgados } \\
\hline Não & $0,54(0,46-0,61)$ & NR & - & - \\
\hline Sim & $0,56(0,53-0,59)$ & 155 & $64,8(62,5-67,0)$ & $46,2(42,3-50,2)$ \\
\hline \multicolumn{5}{|c|}{ Consumo de carnes com gordura } \\
\hline Não & $0,62(0,51-0,72)$ & 220 & $75,1(68,7-80,6)$ & $48,8(34,3-63,5)$ \\
\hline Sim & $0,55(0,53-0,58)$ & 155 & $64,1(61,8-66,3)$ & $46,0(42,3-49,8)$ \\
\hline \multicolumn{5}{|c|}{ Consumo de frango com gordura } \\
\hline Não & $0,57(0,54-0,61)$ & 155 & $68,7(65,7-71,6)$ & $45,1(39,9-50,4)$ \\
\hline Sim & $0,55(0,51-0,58)$ & 155 & $61,3(58,2-64,3)$ & $46,9(41,9-52,0)$ \\
\hline
\end{tabular}

*Classificados como faixa de peso recomendável=1 e Excesso de Peso=0; Sens.: Sensibilidade; Esp.: Especificidade; IC95\%: Intervalo de Confiança a 95\%; PC: Ponto de Corte; NR: Não Recomendado. 
A descrição dos pontos de corte e os níveis de sensibilidade e especificidade dos minutos de AF no lazer, em relação a ausência de excesso de peso corporal são apresentados na Tabela 2 . O ponto de corte com melhor equilíbrio entre sensibilidade e especificidade foi referente aos 150 minutos de AF no lazer para todos os universitários. Esse ponto foi o mesmo observado para os universitários de 35 anos ou mais. Por outro lado, o ponto de corte dos 155 minutos predominou em diferentes características exploratórias (consumo de frutas e hortaliças até quatro dias, consumir salgados, consumir frango com gordura e consumir carne com gordura).

\section{Discussão}

Os minutos de prática de AF em intensidade moderada a vigorosa no lazer apresentou menor poder preditivo, tanto de modo geral quanto para as características exploratórias. O ponto de corte que predominou, entre os demais encontrados foi o de 155 minutos de AF no lazer para os universitários que referiram o consumo de frutas e hortaliças até quatro dias, consumir salgados, consumir frango com gordura e consumir carne com gordura, porém, os 150 minutos de AF no lazer para todos os universitários foi o ponto que melhor apresentou níveis de sensibilidade e especificidade.

Observou-se, nesta pesquisa, tanto de modo geral quanto em relação a determinadas características sociodemográficas e de hábitos alimentares, que os poderes de predição dos minutos de AF no lazer em relação a ausência do excesso de peso corporal atingiram o mínimo recomendado, porém, com menor poder preditivo, pois a variação das áreas das curvas ROC significativas foram de 0,55 a 0,62. Essas áreas foram semelhantes as estimativas dos minutos de $\mathrm{AF}$ moderadas a vigorosas, mensuradas por meio de acelerômetros, para a predição da ausência do excesso de peso corporal em crianças espanholas ${ }^{22}$ e adolescentes de diferentes cidades da Europa $^{23}$. O menor poder preditivo da AF, em relação a ausência de excesso de peso corporal pode ter ocorrido em função da característica do IMC, que representa um índice associado com a adiposidade global ${ }^{24}$, mas, pode ser influenciado pelo volume da massa muscular, que pode ser favorecido via prática de AF no lazer.

Os resultados do presente estudo mostraram que os pontos de corte que mais se repetiram foram os 150 minutos para todos os universitários e para aqueles da maior faixa etária, e os 155 minutos entre os subgrupos (18 a 24 anos, consumo de frutas e hortaliças até quatro dias por semana, consumo de salgados, consumo de carnes com gordura, consumir e não consumir frango com gordura). Esses achados apresentam aproximação com as recomendações de prática de AF de intensidade moderada em todos os domínios (lazer, deslocamento, trabalho e lar), destinadas à prevenção do excesso de peso corporal ${ }^{9-11}$. As diretrizes do ACSM descrevem a quantidade de 150 minutos por semana para prevenir o ganho de peso corporal maior que $3 \%$ e promover a diminuição modesta de dois a três quilogramas ${ }^{9}$.

Nesta pesquisa, o ponto de corte de 150 minutos de AF no lazer para todos os universitários apresentou melhor equilíbrio entre sensibilidade e especificidade. Os níveis de sensibilidade dos minutos de AF no lazer, deste estudo, foram maiores que a especificidade e de forma semelhante, em pesquisa com adolescentes europeus, maior valor de sensibilidade $(79,8 \%)$ do que a especificidade $(26,7 \%)$ foi observado na estimativa dos minutos de AF moderada a vigorosa para a predição da ausência de excesso de peso corporal, porém sem equilíbrio entre essas duas características $^{23}$. Embora as importantes diferenças entre os estudos, quanto as medidas empregadas e o perfil demográfico, neste estudo, para todos os universitários, o equilíbrio entre sensibilidade e especificidade favorece maior estabilidade dos minutos de AF no lazer, contudo, como não foram valores expressivos desta- 
cam a fragilidade para a predição da ausência dessa morbidade.

As limitações deste estudo estão relacionadas ao uso de questionário para o levantamento das informações das AF no lazer, e o uso de medidas referidas da massa corporal e estatura para o cálculo do IMC. No entanto, tanto o instrumento que foi utilizado quanto a estimativa do excesso de peso corporal com base as medidas referidas apresentam níveis satisfatórios de reprodutibilidade e validade concorrente, respectivamente ${ }^{14,15}$. As análises apenas para a AF no tempo livre podem representar uma limitação, em virtude da possibilidade de prática no deslocamento, trabalho e atividades domésticas ${ }^{25}$ e essas práticas tendem a influenciar diretamente no gasto energético total ${ }^{9-11}$.

O não controle das características alimentares nas estimativas de predição da AF no lazer podem ser consideradas como limitação, uma vez que esse comportamento é um dos componentes responsáveis para a manutenção do peso corporal ${ }^{2,9,26}$ e para minimizar essa ausência foram conduzidas análises estratificadas por diferentes hábitos alimentares. Por fim, o delineamento transversal representa a principal limitação desta pesquisa, pois impede o estabelecimento de causalidade, necessário para esta temática, desta forma, o perfil preditivo da $\mathrm{AF}$ no lazer em relação a ausência de excesso de peso corporal implica em cautelas com os resultados observados ${ }^{27}$.

Como pontos fortes do estudo, destaca-se a robustez do procedimento de amostragem na consideração de características vinculadas à instituição, além de procedimento de seleção aleatória simples para o sorteio dos participantes. Soma-se a isso o tempo curto de coleta de dados para evitar o possível viés de resposta devido aos feriados, períodos de aplicação de testes acadêmicos e mudanças climáticas.

Conclui-se que a AF no lazer em intensidade moderada a vigorosa apresentou menor poder para a predição da ausência de excesso de peso corporal em universitários. O ponto de corte com melhor equilíbrio preditivo foram os 150 minutos, contudo, faz-se necessário destacar a necessidade de muita cautela com uso desse ponto de corte, tendo em vista que foram provenientes de estudo transversais. A determinação da quantidade mínima como recomendação de AF para prevenção, controle e/ou tratamento do excesso de peso corporal podem ser melhor estabelecidas por meio de estudos do tipo coorte e experimentais.

\section{Conflito de interesses}

Os autores declaram que não apresentam conflitos.

\section{Contribuição dos autores}

T. F. Sousa (0000-0002-9846-9661) elaborou e coordena o estudo MONISA, realizou as análises estatísticas e a redação do artigo. A. R. Barbosa (0000-0003-09297659) auxiliou na redação e revisão crítica do manuscrito. F. T. Coelho (00000001-5413-5718) auxiliou na redação do manuscrito.

\section{Referências}

1. Low S, Chin MC, Deurenberg-Yap M. Review on epidemic of obesity. Ann Acad Med Singapore. 2009;38(1):57-65.

2. World Health Organization. Global health risks. Mortality and burden of disease attributable to selected major risks. Geneva: WHO, 2009.

3. World Health Organization. Obesity and overweight. Geneva: WHO, 2016. [acessado 2016 jul 05]. Disponível em: <http://www.who.int/mediacentre/factsheets/fs311/en/>.

4. Kelly T, Yang W, Chen CS, Reynolds K, He J. Global burden of obesity in 2005 and projections to 2030. Int J Obesity. 2008;32(9):1431-7.

5. Sousa TF. Excesso de peso corporal em acadêmicos de Educação Física, Bahia. EFDeportes 2010;15(45):1. 
6. Silva DAS, Quadros TMB, Gordia AP, Petroski EL. Associação do sobrepeso com variáveis sócio-demográficas e estilo de vida em universitários. Ciênc Saúde Colet. 2011;16(11):4473-79.

7. Fontes ACD, Pontes LM, Vianna RPT. Prevalência e fatores associados ao sobrepeso e obesidade em estudantes universitários, Paraíba, Brasil. Coleç Pesq Educ Fís. 2012;11(2):149-58.

8. Ainett WSC, Costa VVL, Sá NNB. Fatores associados à insatisfação com a imagem corporal em estudantes de nutrição. RBONE. 2017;11(62):75-8.

9. Donnelly JE, Blair SN, Jakicic JM, Manore MM, Rankin JW, Smith BK, et al. Appropriate physical activity intervention strategies for weight loss and prevention of weight regain for adults. Med Sci Sports Exerc. 2009;41(2):459-71.

10. Haskell WL, Lee IM, Pate RR, Powell KE, Blair SN, Franklin BA, et al. Physical Activity and Public Health: Updated Recommendation for Adults from the American College of Sports Medicine and the American Heart Association. Med Sci Sports Exerc. 2007;39(8):1423-34.

11. World Health Organization. Global recommendations on physical activity for health. Geneva: WHO, 2011.

12. Sousa TF, Fonseca SA, José HPM, Nahas MV. Estudo MONISA: características e aspectos metodológicos. Rev Bras Epidemiol. 2012;15(4): 904-7.

13. Luiz RR, Magnanini MF. A lógica da determinação do tamanho da amostra em investigações epidemiológicas. Cad Saúde Colet. 2000;8(2):9-28.

14. Sousa TF, Fonseca SA, José HPM, Nahas MV. Validade e reprodutibilidade do questionário Indicadores de Saúde e Qualidade de Vida de Acadêmicos (Isaq-A). Arq Ciênc Esporte. 2013;1(1):21-30.

15. Sousa TF, Barbosa AR. Validade das medidas referidas da massa corporal e estatura em universitários. ABCS Health Sciences. 2016;41(2):71-7.

16. World Health Organization. Obesity: preventing and managing the Global Epidemic. Report on a WHO Expert Consultation on Obesity, 3-5 June 1997. Geneva: OMS, 1997.

17. Ainsworth BE, Haskell WL, Herrmann SD, Meckes N, Bassett DR Jr, Tudor-Locke C, et al. 2011 Compendium of physical activities: a second update of codes and MET values. Med Sci Sports Exerc. 2011;43(8):1575-81.

18. Hallal PC, Victora CG, Wells JCK, Lima RC. Physical inactivity: prevalence and associated variables in Brazilian adults. Med Sci Sports Exerc. 2003;35(11):1894-900.

19. Sousa TF, Nahas MV. Prevalência e fatores associados a menores níveis de prática de atividades físicas no lazer em estudantes de uma universidade pública do Estado da Bahia. Rev Bras Ativ Fís Saúde. 2011;16(4):322-9.

20. Ministério da Saúde do Brasil. Vigilância de Fatores de Risco e Proteção para Doenças Crônicas por Inquérito Telefônico: estimativas sobre frequência e distribuição sóciodemográfica de fatores de risco e proteção para doenças crônicas nas capitais dos 26 Estados brasileiros e no Distrito Federal em 2006. Brasília, Ministério da Saúde, 2007. 90 p.

21. Lasko TA, Bhagwat JG, Zou KH, Ohno-Machado L. The use of receiver operating characteristic curves in biomedical informatics. J Biomed Inform. 2005;38(5):404-15.

22. Laguna M, Ruiz JR, Lara MT, Aznar S. Recommended levels of physical activity to avoid adiposity in Spanish children. Pediatr Obes. 2013;8(1):62-9.

23. Martinez-Gomes D, Ruiz JR, Ortega FB, Veiga OL, Moliner-Urdiales D, Mauro B, et al. Recommended Levels of Physical Activity to Avoid an Excess of Body Fat in European Adolescents. The HELENA Study. Am J Prev Med. 2010;39(3):203-11.

24. Costa RF, Guiselini M, Fisberg M. Correlação entre porcentagem de gordura e índice de massa corporal de freqüentadores de academia de ginástica. Rev Bras Cienc Mov. 2007;15(4):39-46.

25. Florindo AA, Hallal PC, Moura EC, Malta DC. Prática de atividades físicas e fatores associados em adultos, Brasil, 2006. Rev Saúde Pública. 2009;43(Supl 2):65-73.

26. Gigante DP, Minten GC, Horta BL, Barros FC, Victora CG. Avaliação nutricional de adultos da coorte de nascimentos de 1982, Pelotas, RS. Rev Saúde Pública. 2008;42(Supl. 2):60-9.

27. Ahrens W, Pigeout I. Handbook of Epidemiology. Germany: Springer, 2005.

Endereço para

Correspondência

Thiago Ferreira de Sousa

tfsousa_thiago@yahoo.com.br
Universidade Federal do Recôncavo

da Bahia. Centro de Formação de

Professores. Avenida Nestor de Mello

Pita, n 535 - Número da caixa postal:

64, Centro - Amargosa, BA, Brasil,

CEP: $45.300-000$.
Recebido

Revisado

05/07/2016

$18 / 10 / 2016$

$21 / 02 / 2017$

$08 / 05 / 2017$

$08 / 05 / 2017$ 